\title{
COMPUTATIONAL ASPECTS \\ OF SOAP BUBBLE DEFORMATIONS
}

\author{
U. PINKALL AND I. STERLING
}

(Communicated by Jonathan M. Rosenberg)

\begin{abstract}
H$-surfaces are studied via soliton theory with the aid of a computer.
\end{abstract}

We search for the simplest possible one-parameter family of constant mean curvature (CMC) tori. The deformations presented here are given by a closed one-dimensional orbit in the moduli space and are distinguished by having one family of spherical curvature lines. Such CMC tori have been studied by Wente $[3,4]$ based on a method due to Dobriner [1].

As in [2] let $F: \mathbb{R}^{2} \rightarrow \mathbb{R}^{3}$ be a conformal parametrization with torsion invariant $E=e^{i \alpha}$ of a surface with CMC $H=\frac{1}{2}$. Define $\omega: \mathbb{R}^{2} \rightarrow \mathbb{R}$ by $\left\langle F_{z}, F_{\bar{z}}\right\rangle=2 e^{2 \omega}$, and let $N: \mathbb{R}^{2} \rightarrow \mathbb{R}^{3}$ be a unit normal vector field for $F$. Then

(1)

$$
\left(\begin{array}{c}
F \\
F_{z} \\
F_{\bar{z}} \\
N
\end{array}\right)_{z}=\left(\begin{array}{c}
F_{z} \\
2 \omega_{z} F_{z}-E N \\
e^{2 \omega} N \\
\frac{1}{2}\left[-F_{z}+E e^{-2 \omega} F_{\bar{z}}\right]
\end{array}\right),
$$

$$
\left(\begin{array}{c}
F \\
F_{z} \\
F_{\bar{z}} \\
N
\end{array}\right)_{\bar{z}}=\left(\begin{array}{c}
F_{\bar{z}} \\
e^{2 \omega} N \\
2 \omega_{\bar{z}} F_{\bar{z}}-\bar{E} N \\
\frac{1}{2}\left[-F_{z}+\bar{E} e^{-2 \omega} F_{z}\right]
\end{array}\right),
$$

where, for example,

$$
\begin{gathered}
F(0,0)=(0,0,0), \quad F_{z}(0,0)=e^{\omega(0,0)}(1, i, 0), \\
F_{\bar{z}}(0,0)=e^{\omega(0,0)}(1,-i, 0), \quad \text { and } \quad N(0,0)=(0,0,1) .
\end{gathered}
$$

In [2] we define CMC surfaces of finite type and prove that they come from

Received by the editors July 17, 1991 .

1991 Mathematics Subject Classification. Primary 53A05; Secondary 35Q20. 
solutions of an ODE-system. For type 2 surfaces we have

$$
\begin{aligned}
& \left(\begin{array}{c}
\omega \\
u_{1} \\
u_{1 z} \\
u_{2} \\
v
\end{array}\right)_{z}=\left(\begin{array}{c}
u_{1} \\
u_{1 z} \\
u_{2}+2 u_{1} \varphi_{1} \\
s \operatorname{Re} v+c i \operatorname{Im} v-a_{2} u_{1 z} \\
u_{1} v+e^{-\omega}\left(\bar{a}_{2} \bar{u}_{1}+\bar{u}_{2}\right)
\end{array}\right), \\
& \left(\begin{array}{c}
\omega \\
u_{1} \\
u_{1 z} \\
u_{2} \\
v
\end{array}\right)_{\bar{z}}=\left(\begin{array}{c}
-.5 S \\
-C u_{1} \\
S \varphi_{1}-C u_{1 z} \\
-\bar{u}_{1} v-e^{\omega}\left(a_{2} u_{1}+u_{2}\right)
\end{array}\right),
\end{aligned}
$$

with $s=\sinh \omega, c=\cosh \omega, S=\sinh 2 \omega, C=\cosh 2 \omega, u_{1}=\omega_{z}, \varphi_{1}=u_{1}^{2}$, and $u_{2}=\omega_{z z z}-2 \omega_{z}^{3}$.

The corresponding surface twists around an axis (see [2] for details) whose direction is given by the vector

$$
J=\tilde{f} N+\frac{1}{2} \tilde{g} F_{z}+\frac{1}{2} \tilde{h} F_{\bar{z}} .
$$

A doubly periodic surface is a torus if both periods have translation zero along the axis and rotate around the axis by a rational multiple of $\pi$.

The dependence of this axis on the torsion parameter $E$ yields the spectral curve $M$ via

$$
P=\sum_{i=0}^{8} c_{i} E^{i}
$$

Now let $\mathbb{C}^{4^{*}}$ be the complex vector space of holomorphic 1 -forms on $M$ with standard basis $\eta_{1}, \ldots, \eta_{4}$,

$$
\eta_{i}=E^{i-1} d E / \sqrt{E P(E)} \text {. }
$$

The $A$ matrix is defined by

$$
A_{i j}=\oint_{a_{j}} \eta_{i}, \quad 1 \leq i, j \leq 4, a_{1}, \ldots, a_{4} \text { as in Figure } 1 .
$$

The periodicity conditions are

$$
\sum_{j=1}^{4} n_{j} A_{i j}=0 \quad \text { and } \quad \sum_{j=1}^{4} m_{j} A_{i j}=0 \quad \text { for } i=2,3,
$$

which if satisfied give the lattice generators

$$
w_{1}=\sum_{j=1}^{4} n_{j} A_{4 j}=0, \quad w_{2}=\sum_{j=1}^{4} m_{j} A_{4 j}=0 .
$$

Wente, following Dobriner, shows that $F$ has one family of spherical curvature lines if and only if

$$
\omega_{y} \omega_{x y y}-\omega_{x y} \omega_{y y}-\omega_{x} \omega_{y}^{3}=0 .
$$

By a lengthy though direct calculation this yields the following restrictions on equations (1) and (2). (In complex coordinates (9) is $-i(f-\bar{f})=0$ where $f=\omega_{z} \omega_{z z z}+\omega_{z} \omega_{\overline{z z z}}+C \omega_{z}^{2}-\omega_{z z}^{2}-S \omega_{z z}-\omega_{z}^{4}-2 \omega_{z} \omega_{\bar{z}}^{3}$. It follows that $u_{2}-\bar{u}_{2}+k u_{1}-k \bar{u}_{1}=0$. The normal form (see $\left.[2, \S 4]\right)$ is $-u_{3}=(k+1) u_{2}-$ $\left.\bar{u}_{2}+a_{1} u_{1}-(k+1) \bar{u}_{1}.\right)$ 


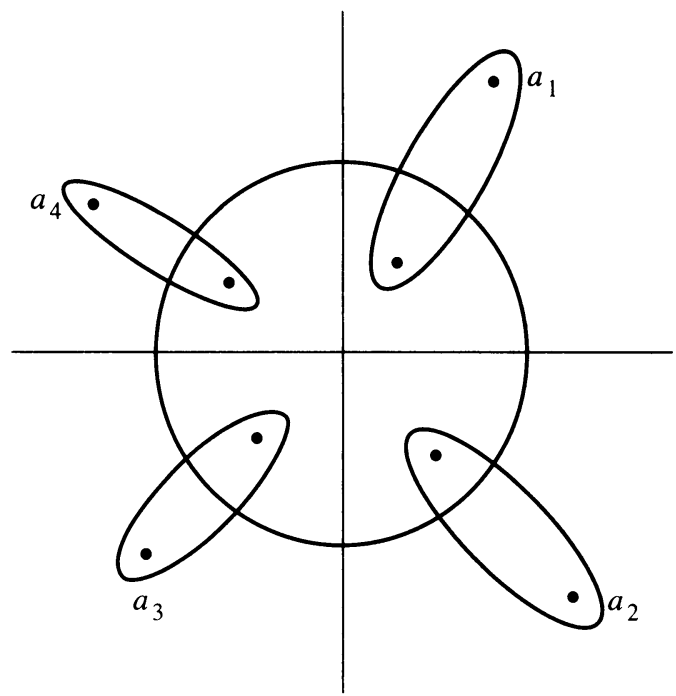

FIGURE 1

Proposition 3.1. $A$ CMC surface with one family of spherical curvature lines is of type 2 and satisfies (for some constant $k$ )

(i) $E=1$,

(ii) $u_{1 z}(0,0) \in \mathbb{R}$,

(iii) $u_{2}(0,0) \in \mathbb{R}$,

(iv) $a_{2}=k+1 \in \mathbb{R}$,

(v) $u_{2 z}(0,0)=(-k-C(0,0)) u_{1 z}(0,0)-\frac{k}{2} S(0,0)$.

In summary (see [3] for details), we input four reals, namely, $\omega, u_{1 z}, u_{2}, a_{2}$. In one direction $\omega$ is periodic; in the other it is quasiperiodic. This quasiperiodicity is measured by a single angle and, hence, gives one real periodicity condition. Since one family of curvature lines lie on spheres, one translational period is zero, and since we have a plane of reflection, one rotation is zero; therefore we have only two closing conditions. This yields one free real parameter.

We now give two ways to compute the one-dimensional orbit of CMC tori corresponding to one that has been found. Finding this orbit (which we think of as lying in the initial condition space) is easy since we only need to find those initial conditions which leave the coefficients of the spectral curve unchanged.

Method 1. Suppose $a_{2}, \omega, u_{1 z}, u_{2}$ correspond to a closed Dobriner-Wente torus. The relevant coefficients of $P$ are

$$
\begin{aligned}
& c_{6}=4 S u_{1 z}+2\left(a_{2}-1\right)(C+1)-4 u_{1 z}^{2}+a_{2}^{2}, \\
& c_{5}=8(C-1) u_{1 z}^{2}+8 a_{2} S u_{1 z}+2 a_{2}^{2}(C+1)+4 u_{2}^{2}-2 a_{2} .
\end{aligned}
$$

With the notation

$$
\begin{gathered}
2 \alpha=a_{2}-1, \quad 4 \beta=a_{2}^{2}+2\left(a_{2}-1\right)-c_{6}, \\
4 \gamma=2 a_{2}\left(a_{2}-1\right)-c_{5}, \quad u=u_{1 z}, \quad t=u_{2},
\end{gathered}
$$




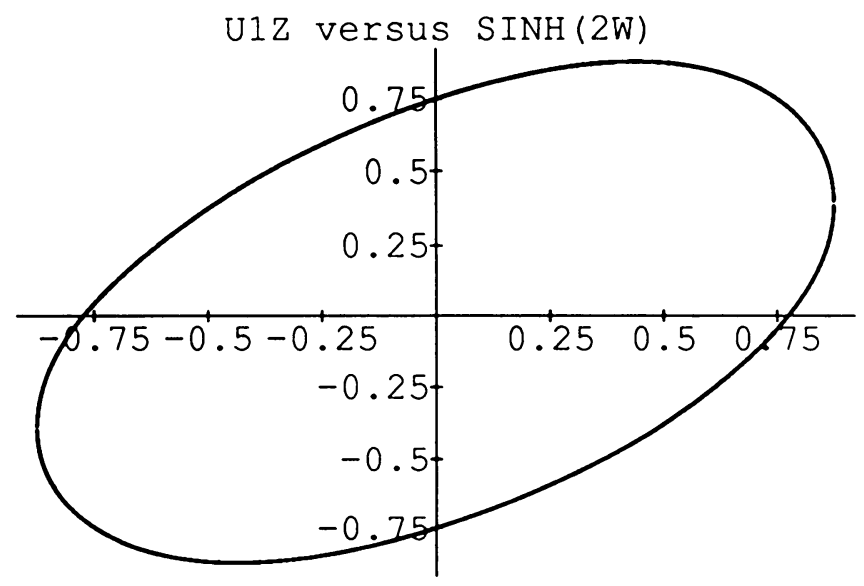

FIGURE 2

(10) can be rewritten as

$$
\begin{gathered}
u(S-u)+\alpha C+\beta=0, \\
t^{2}=-\left[2(C-1) u^{2}+2(2 \alpha+1) S u+\frac{1}{2}(2 \alpha+1)^{2} C+\gamma\right] .
\end{gathered}
$$

A short computation shows that the first equation in (12) is equivalent to the quadratic equation

$$
0=\left[\alpha^{2}-u^{2}\right] S^{2}+2 u\left[u^{2}-\beta\right] S+\left[\alpha^{2}-\left(u^{2}-\beta\right)^{2}\right] .
$$

The projection of solutions to (13) as $u$ runs from minus infinity to infinity is as shown in Figure 2.

Method 2. Letting $c_{5}=f\left(\omega, u_{1 z}, u_{2}\right)$ and $c_{6}=g\left(\omega, u_{1 z}, u_{2}\right)$ and recalling that $a_{2}$ is an invariant, we see that the orbit is a curve in $\left(\omega, u_{1 z}, u_{2}\right)$-space with tangent vector $T=\operatorname{grad} f \times \operatorname{grad} g$. Therefore, given a point on the orbit, one may numerically find the orbit recursively by integrating the vector field $T$.

So we are looking for circles in four space, which using either Method 1 or 2 can be completely recovered once we find one point on them. It therefore suffices to check three-dimensional slices. We consider the slice $u_{2}(0,0)=0$. Geometrically this corresponds to the images in 3-space of both coordinate axes in $\mathbb{R}^{2}$ being planar.

In practice we input $a_{2}, \omega(0,0), u_{1 z}(0,0)$ with the mouse, and from each input we have many possible output windows, for example, the spectral curve together with the cycles $a_{i}$ (as shown in Figure 1). The orbit projected to an appropriate two-plane in the initial condition space is shown in Figure 2. These are helpful for understanding the relationships between the orbits in the moduli space and properties of the spectral curves.

The most important output for finding closed tori or other interesting examples are the images of the coordinate axes in $\mathbb{R}^{2}$ which are now both planar lines of curvature: one in the $X-Z$ plane, which may be quasiperiodic, and one in the $Y-Z$ plane, which is always periodic. By observing $F\left(k_{1} w_{1}\right)$ and $F\left(k_{2} w_{2}\right)$ for large enough integer multiples of $w_{1}$ and $w_{2}$, one can check closing conditions (does the curve come back to itself); see Figure 3. All this can be done with low precision. Once you get near a closed surface you can go to a 


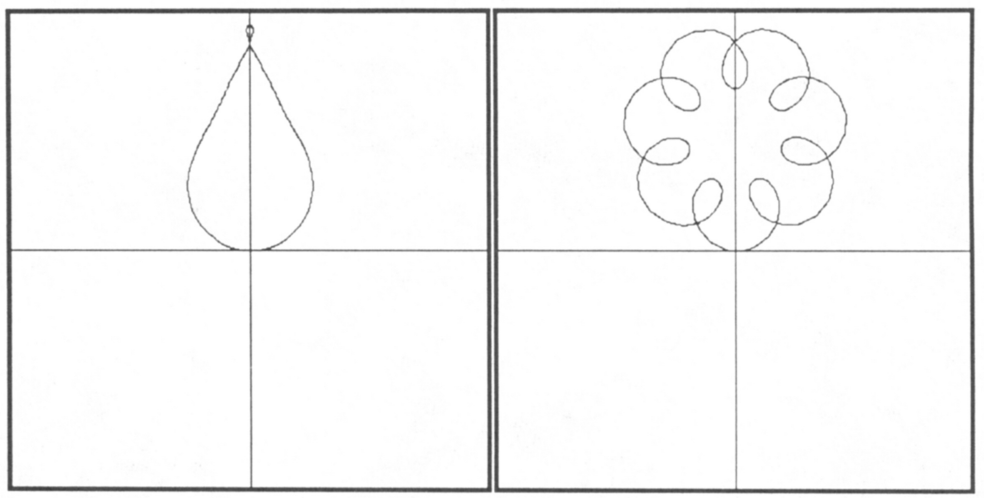

FIGURE 3


FIGURE 4 

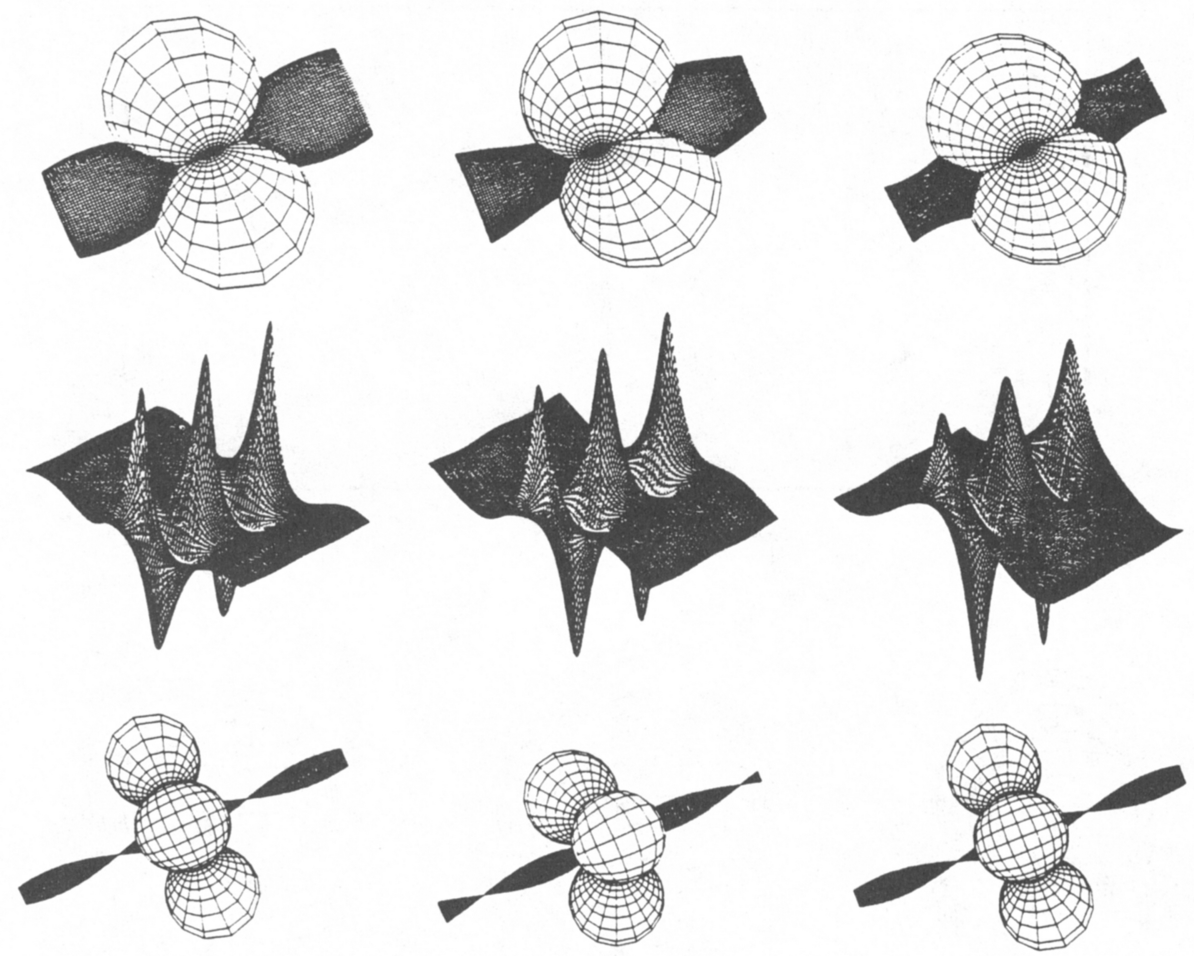

FIGURE 5

root finder (Newton method), which takes you to the surface with "any" desired accuracy.

Using these methods we found the seven-lobed tori shown (together with their metrics $\omega(x, y)$ and their parallel surfaces with $K=1)$ deforming in Figure 4 and the deforming soliton-like cylinders with embedded Delaunay ends shown in Figure 5.

\section{ACKNOWLEDGMENT}

We would like to thank U. Abresch and H. Wente for interesting discussions.

\section{REFERENCES}

1. H. Dobriner, Die Flächen constanter Krümmung mit einem System sphärischer Krümmunglinien dargestell mit Hilfe von Thetafunktionen zweier Variabeln, Acta Math. 9 (1886), 73-104.

2. U. Pinkall and I. Sterling, On the classification of constant mean curvature tori, Ann. of Math. (2) 130 (1989), 407-451.

3. H. Wente, Constant mean curvature immersions with one family of curvature lines spherical, preprint No. 10, SFB 256, Bonn, 1988.

4. __ Constant mean curvature immersions of Enneper type, Mem. Amer. Math. Soc., vol. 100, Amer. Math. Soc., Providence, RI, 1992.

Fachbereich Mathematik, Technische Universität Berlin, Strasse des 17.Juni 135, D-1000 BerLin 12, Germany

E-mail address, U. Pinkall: ulrich@willmore.math.tu-berlin.de

Department of Mathematics, University of Toledo, Toledo, OHio 43606

E-mail address, I. Sterling: isterli@uoft02.utoledo.edu 